Pure and Applied Mathematics Quarterly

Volume 2, Number 3

(Special Issue: In honor of

Robert MacPherson, Part 1 of 3)

$637-653,2006$

\title{
Convexity Theorems for Varieties Invariant under a Borel Subgroup
}

\author{
Victor Guillemin and Reyer Sjamaar \\ To Bob MacPherson, our teacher and friend
}

\begin{abstract}
Atiyah proved that the moment map image of the closure of an orbit of a complex torus action is convex. Brion generalized this result to actions of a complex reductive group. We extend their results to actions of a maximal solvable subgroup.
\end{abstract}

\section{Contents}

Introduction 637

1. Convexity results of Atiyah and Brion 638

2. Convexity theorems for $B$-varieties 641

3. The norm of an $N$-invariant holomorphic section 644

4. Denseness of the rational points 648

5. "Schubert" varieties 650

References $\quad 652$

\section{INTRODUCTION}

Let $M$ be a compact Kähler manifold and $L$ a Hermitian line bundle over $M$ whose curvature form is the Kähler form. In addition, let $G$ be a compact Lie group and $\tau$ a Kählerian action of $G$ on $(M, L)$. From this action one gets a moment map $\Phi: M \rightarrow \mathfrak{g}^{*}$ and a holomorphic action $\tau_{\mathbf{C}}$ of the complexified Lie

Received April 29, 2005.

2000 Mathematics Subject Classification. 53D20 (14L24 53C55) 
group $G_{\mathbf{C}}$ on $(M, L)$. In particular, any Borel subgroup $B$ of $G_{\mathbf{C}}$ acts on $M$ and $L$, and the main goal of this paper is to prove a Kirwan type convexity theorem for the moment images of $B$-invariant subvarieties of $M$. Let $T$ be the maximal torus of $G$ contained in $B$ and let $\mathfrak{t}_{+}^{*}$ be the closed Weyl chamber in $\mathfrak{t}^{*}$ which is positive with respect to $B$. Our main result, which is described in more detail in Section 2, asserts

Theorem. If $X$ is a $B$-invariant irreducible closed analytic subvariety of $M$, the moment image of $X$ in $\mathfrak{g}^{*}$ intersects $\mathfrak{t}_{+}^{*}$ in a convex polytope.

This result is a $B$-analogue of well-known convexity theorems of Atiyah and Brion, which we will review in Section 1, partly as a motivation for our result and partly because the techniques used by Brion to prove his $G_{\mathbf{C}}$-convexity theorem will be used in our proof as well.

One other major ingredient in our proof is a $B$-analogue of a fundamental result in geometric invariant theory. This $B$-analogue asserts that if $s$ is a global holomorphic section of $L$ which is an eigensection for the action of $B$ and which does not vanish at a point $x$, then the norm of $s$, restricted to the closure of the $B$-orbit through $x$, takes its maximum at a point $y$ such that $\Phi(y)$ is in the chamber $\mathfrak{t}_{+}^{*}$. This theorem and variants of it will be discussed in Section 3 . The final ingredient of our proof, a rationality statement concerning the moment image, is explained in Section 4.

Our main result has some interesting applications to "complex Morse theory". It is well known that a generic component of the moment map is a Morse-Bott function, and Carrell and Sommese have shown that the unstable manifolds of this Morse-Bott function are complex submanifolds and that their closures are analytic subvarieties. Moreover, as we show in Section 5, for a "dominant" choice of a moment map component these varieties are $B$-invariant. (For instance, for a flag variety the unstable manifolds are the Bruhat cells: the $B$-orbits.) Hence their moment images intersected with $\mathfrak{t}_{+}^{*}$ are convex polytopes. In other words, the Morse-Bruhat-Carrell-Sommese decomposition of $M$ gets reflected in a "stratification" of the Kirwan polytope.

\section{Convexity Results of Atiyah and Brion}

The main result of this paper is a nonabelian generalization of a theorem about torus actions proved by Atiyah in [1]. Let $(M, \omega)$ be a compact Kähler manifold and $\tau$ a Kählerian action of the torus $T=\left(S^{1}\right)^{n}$ on $M$. From $\tau$ one gets a holomorphic action $\tau_{\mathbf{C}}$ of the complex torus $T_{\mathbf{C}}=\left(\mathbf{C}^{*}\right)^{n}$. If $\tau$ has fixed points, there is a moment map $\Phi: M \rightarrow \mathfrak{t}^{*}$, and Atiyah's theorem asserts:

1.1. Theorem. Let $X=\overline{T_{\mathbf{C}} x}$ be the closure of the $T_{\mathbf{C}}$-orbit through a point $x \in M$. Then the moment image of $X$ is a convex polytope. More explicitly, 
$\Phi(X)$ is the convex hull of the set $\Phi\left(X^{T}\right)$, where $X^{T}$ denotes the set of fixed points of $\tau \mid X$.

If $\omega$ is the curvature form of a $T$-equivariant Hermitian holomorphic line bundle $L$ on $M$, there is an alternative description of $\Phi(X)$. The space of global holomorphic sections $\Gamma(M, L)$ decomposes under the action of $T$ into a direct sum of weight spaces, $\Gamma(M, L)=\bigoplus_{\lambda \in \Lambda} \Gamma(M, L)_{\lambda}$, where $\Lambda=\operatorname{Hom}(T, \mathbf{U}(1))$ is the weight lattice of $T$. (As usual, we will identify $\Lambda$ with a subgroup of $\mathfrak{t}^{*}$ by identifying the weight $\lambda$ with the functional $T_{1} \lambda /(2 \pi \sqrt{-1})$.) Atiyah's description of $\Phi(X)$ is equivalent to:

1.2. Theorem. Let $x \in M$ and let $X=\overline{T_{\mathbf{C}} x}$. Suppose $L$ is generated by its global sections. Then $\Phi(X)$ is the convex hull of the subset

$$
\left\{\lambda \in \Lambda \mid \exists s \in \Gamma(M, L)_{\lambda}, s(x) \neq 0\right\}
$$

of $\mathfrak{t}^{*}$.

It is this version of Atiyah's theorem which we'll generalize below. First, however, we mention some of its consequences.

1.3. Corollary. Let $X$ be an arbitrary $T$-invariant irreducible closed subvariety of $M$ and let $i: X \rightarrow M$ be the inclusion map.

(i) $\Phi(X)$ is the convex hull in $\mathfrak{t}^{*}$ of the set

$$
\left\{\lambda \in \Lambda \mid \exists s \in \Gamma(M, L)_{\lambda}, i^{*} s \neq 0\right\} .
$$

(ii) $\Phi(X)$ is the convex hull in $\mathfrak{t}^{*}$ of the set $\Phi\left(X^{T}\right)$.

(iii) $\Phi(X)$ is an integral convex polytope.

(iv) The set consisting of all $x$ in $X$ for which $\Phi\left(\overline{T_{\mathbf{C}} x}\right)=\Phi(X)$ is nonempty and Zariski open in $X$.

(v) The collection of polytopes $\Phi(X)$, where $X$ ranges over all $T_{\mathbf{C}}$-invariant irreducible closed subvarieties of $M$, is finite.

A special case of Corollary 1.3 is a convexity theorem of Atiyah for Schubert varieties. Suppose $T$ is a Cartan subgroup of a connected compact Lie group, $G$. Let $G_{\mathbf{C}}$ be the complexification of $G$, let $B \supseteq T$ be a Borel subgroup of $G_{\mathbf{C}}$ and let $M$ be the full flag variety $G_{\mathbf{C}} / B$. For any $\mu \in \Lambda$ let $\mathbf{C}_{\mu}$ be the one-dimensional $B$-module with weight $\mu$ and let $L_{\mu}=G_{\mathbf{C}} \times{ }^{B} \mathbf{C}_{\mu}$ be the corresponding $G_{\mathbf{C}}$-homogeneous line bundle over $M$. The curvature form $\omega_{\mu}$ (with respect to the $G$-invariant Hermitian metric on $L_{\mu}$ defined by the absolute value on $\mathbf{C}$ ) is nondegenerate if and only if the weight $\mu$ is regular. It is well known that $\omega_{\mu}$ is Kähler if and only if $\mu \in-$ Int $\mathfrak{t}_{+}^{*}$, where $\mathfrak{t}_{+}^{*}$ is the closed Weyl chamber in $\mathfrak{t}^{*}$ which is positive with respect to $B$. A moment map $\Phi_{\mu}$ for the $G$-action is found by composing the maps

$$
M=G_{\mathbf{C}} / B \longrightarrow G / T \longrightarrow \mathfrak{g}^{*},
$$


where the first map is the inverse of the diffeomorphism $G / T \rightarrow M$ induced by the inclusion $G \rightarrow G_{\mathbf{C}}$ and the second map is defined by $g T \mapsto g \mu$. In fact, $\Phi_{\mu}$ is a $G$-equivariant symplectomorphism from $M$ onto the coadjoint orbit through $\mu$. Let $W=N_{G}(T) / T$ be the Weyl group of $(G, T)$. The $T$-fixed points in $M$ are precisely the cosets $w B$ with $w \in W$. Every orbit of the left $B$-action on $M$ passes through a unique $T$-fixed point. The Schubert variety $X_{w}$ is by definition the closure of the $B$-orbit through the $T$-fixed point $w B$. The Bruhat order on $W$ is defined by $v \leq w$ if and only if $X_{v} \subseteq X_{w}$. Thus $\left(X_{w}\right)^{T}=\{v B \mid v \leq w\}$. Since the $T$-moment map $M \rightarrow \mathfrak{t}^{*}$ is just the composition of $\Phi_{\mu}$ and the projection $\mathfrak{g}^{*} \rightarrow \mathfrak{t}^{*}$, one obtains from Corollary 1.3(ii):

1.4. Theorem ([1]). The projection onto $\mathfrak{t}^{*}$ of the Schubert variety $X_{w}$ is equal to the convex hull of the set $\{v \mu \mid v \leq w\}$.

Theorem 1.2 and its various corollaries have been generalized by Brion to nonabelian groups in the setting of complex projective varieties. Namely let $G$, $T$ and $B$ be as above and let $N=[B, B]$ be the unipotent radical of $B$. Let $\tau$ be an action of $G$ on $\mathbf{C P}^{n}$ coming from a unitary representation of $G$ on $\mathbf{C}^{n+1}$. Then $G$ acts on the polynomial algebra $S=\bigoplus_{r \geq 0} S_{r}$, where $S_{r}=\Gamma\left(\mathbf{C P}^{n}, L^{r}\right)$ and $L=\mathcal{O}(1)$ is the canonical hyperplane bundle. Let $\Lambda_{+}=\Lambda \cap \mathfrak{t}_{+}^{*}$ be the semigroup of dominant weights of $(G, T)$. For $\lambda \in \Lambda_{+}$and $r \in \mathbf{N}$ let $S_{\lambda, r}$ be the isotypical $G$-submodule of $S_{r}$ of highest weight $\lambda$. The set $S^{N}$ consisting of all $N$-invariant polynomials is a subalgebra of $S$ which is preserved by the action of $T$. Let us put $S_{\lambda, r}^{N}=\left(S_{\lambda, r}\right)^{N} ;$ then

$$
S^{N}=\bigoplus\left\{S_{\lambda, r}^{N} \mid(\lambda, r) \in \Lambda_{+} \times \mathbf{N}\right\}
$$

is a bigrading of the algebra $S^{N}$ by weight and degree. Let $X$ be any $G_{\mathbf{C}^{-} \text {-invariant }}$ irreducible closed subvariety $X$ of $\mathbf{C P}^{n}$ and let $I(X)$ be the homogeneous ideal of $S$ consisting of all polynomials vanishing on $X$. Then $I(X)^{N}$ is a homogeneous ideal of $S^{N}$ and the quotient algebra $A(X)=S^{N} / I(X)^{N}$ is $\Lambda_{+} \times \mathbf{N}$-graded. Brion defines

$$
\mathcal{C}(X)=\left\{\lambda \in \Lambda \otimes \mathbf{Q} \mid A(X)_{r \lambda, r} \neq 0 \text { for some } r>0\right\} .
$$

In other words, a rational weight $\lambda \in \Lambda \otimes \mathbf{Q}$ is in $\mathcal{C}(X)$ if and only if there exist a positive integer $r$ such that $r \lambda$ is integral and a section $s \in S_{r \lambda, r}^{N}$ such that $s$ does not vanish on $X$. The set $\mathcal{C}(X)$ is contained in the intersection of the chamber $\mathfrak{t}_{+}^{*}$ with the $\mathbf{Q}$-vector space $\Lambda \otimes \mathbf{Q}$. The assumption that $X$ is irreducible implies that $A(X)$ has no zero divisors and hence that $\mathcal{C}(X)$ is convex over the rationals. A theorem of Hadziev and Grosshans (see e.g. [15, Kapitel III.3.2] says that the algebra $S^{N}$ is finitely generated. Since $A(X)$ is the image of the map $S^{N} \rightarrow(S / I(X))^{N}$ induced by the quotient map $S \rightarrow S / I(X)$, $A(X)$ is likewise finitely generated. By selecting a finite system of homogeneous generators $\left\{a_{i} \in A(X)_{\lambda_{i}, r_{i}} \mid i=1,2, \ldots, k\right\}$, one sees immediately that $\mathcal{C}(X)$ is 
the convex hull of the rational weights $\lambda_{1} / r_{1}, \lambda_{2} / r_{2}, \ldots, \lambda_{k} / r_{k}$. Thus $\mathcal{C}(X)$ is a convex polytope over $\mathbf{Q}$. Now let $\Phi: \mathbf{C P}^{n} \rightarrow \mathfrak{g}^{*}$ be the moment map for the action of $G$ on $\mathbf{C P}^{n}$. Brion proves:

1.5. Theorem ([4]). Let $X$ be a $G_{\mathbf{C}}$-invariant irreducible closed subvariety of $\mathbf{C P}^{n}$. Then the intersection $\Delta(X)=\Phi(X) \cap \mathfrak{t}_{+}^{*}$ is equal to the closure of $\mathcal{C}(X)$, and $\mathcal{C}(X)$ is equal to the set of rational points in $\Delta(X)$. Hence $\Delta(X)$ is a rational convex polytope.

(This result builds on prior work by Ness and Mumford [16]; cf. also Guillemin and Sternberg [11].) In particular, for every $x \in \mathbf{C P}^{n}$ the set $\Delta\left(\overline{G_{\mathbf{C}} x}\right)$ is a rational convex polytope. Brion also proves nonabelian analogues of the other assertions in Corollary 1.3. (However, in contrast to the abelian case, $\Delta$ is not necessarily an integral polytope. This is why the powers of $L$ must be incorporated in the definition of the set $\mathcal{C}(X)$.)

\section{CONVEXity THEOREMS FOR $B$-VARIETIES}

Our main observation is that these results of Brion are also true for $B$-invariant subvarieties of $G_{\mathbf{C}}$-manifolds. Namely let $M$ be a compact complex manifold, $L \rightarrow M$ a positive Hermitian holomorphic line bundle with Hermitian connection $\nabla$ and curvature form $\omega$, and $\tau$ an action of $G$ on $L$ by line bundle automorphisms which preserve the complex structure on $M$ and the Hermitian structure on $L$. The group of all line bundle automorphisms which preserve the holomorphic structure on $L$ is a complex Lie group; and therefore the $G$-action extends uniquely to a $G_{\mathrm{C}}$-action by holomorphic line bundle automorphisms on $L$. Moreover, since the action of $G$ on $L$ preserves its Hermitian structure, the action of $G$ on $M$ preserves $\omega$. The space $C^{\infty}(M, L)$ of all smooth sections of $L$ is a $G$-module in a natural way. Let $\mathcal{L}(\xi) s$ denote the Lie derivative of a section $s \in C^{\infty}(M, L)$ along $\xi \in \mathfrak{g}$. As observed by Kostant [14, Theorem 4.3.1], the first-order operators $\nabla\left(\xi_{M}\right)$ and $\mathcal{L}(\xi)$ have the same principal symbol, and the zeroth order operator $\mathcal{L}(\xi)-\nabla\left(\xi_{M}\right)$ is given by multiplication by an imaginary-valued function. The map $\phi: \mathfrak{g} \rightarrow C^{\infty}(M, \mathbf{R})$ defined by

$$
\phi(\xi)=\frac{1}{2 \pi \sqrt{-1}}\left(\mathcal{L}(\xi)-\nabla\left(\xi_{M}\right)\right)
$$

satisfies $d \phi(\xi)=\iota\left(\xi_{M}\right) \omega$ and is $G$-equivariant. Therefore the map $\Phi: M \rightarrow \mathfrak{g}^{*}$ defined by $\langle\Phi, \xi\rangle=\phi(\xi)$ is a moment map for the $G$-action on $M$.

As in Section 1 we consider the algebra of sections $S=\bigoplus_{r \geq 0} \Gamma\left(M, L^{r}\right)$ and its subalgebra $S^{N}$, which has a grading

$$
S^{N}=\bigoplus\left\{S_{\lambda, r}^{N} \mid(\lambda, r) \in \Lambda_{+} \times \mathbf{N}\right\} .
$$


We introduce, for any $B$-invariant irreducible closed analytic subvariety $X$ of $M$, the homogeneous ideal $I(X)$ of all sections vanishing on $X$, the quotient algebra $A(X)=S^{N} / I(X)^{N}$, and the set of rational weights

$$
\mathcal{C}(X)=\left\{\lambda \in \Lambda \otimes \mathbf{Q} \mid A(X)_{r \lambda, r} \neq 0 \text { for some } r>0\right\} .
$$

(Sometimes we shall write $\mathcal{C}(X, L)$ instead of $\mathcal{C}(X)$ to emphasize the dependence of this set on the linearization $L$.) As before, $\mathcal{C}(X)$ is contained in the chamber $\mathfrak{t}_{+}^{*}$ and is the convex hull of a finite subset of $\Lambda \otimes \mathbf{Q}$. Now define $\Delta(X)=\Phi(X) \cap \mathfrak{t}_{+}^{*}$. In Sections 3 and 4 we will prove the following assertion, which is the main result of this paper.

2.1. Theorem. Let $X$ be a B-invariant irreducible closed subvariety of $M$. Then $\Delta(X)$ is equal to the closure of $\mathcal{C}(X)$, and $\mathcal{C}(X)$ is equal to the set of rational points in $\Delta(X)$. Hence $\Delta(X)$ is a rational convex polytope.

2.2. Remarks. (i) By Kodaira's embedding theorem, $M$ embeds holomorphically into the projective space associated to $\Gamma\left(M, L^{r}\right)^{*}$ for sufficiently large $r$. It is easy to see that the Kodaira map is $G_{\mathbf{C}}$-equivariant. Therefore $M$ is a projective $G_{\mathbf{C}}$-variety. However, the Kodaira map is rarely symplectic. Thus the symplectic form $\omega$ is usually not the restriction of the Fubini-Study form, nor is the moment map $\Phi$ usually the restriction of the standard moment map on projective space.

(ii) Here is a heuristic argument in favour of Theorem 2.1. Let $M_{\mathrm{impl}}$ be the imploded cross-section of $M$. This is a certain stratified symplectic space with a Hamiltonian $T$-action on the strata. (See [9].) It can be identified with Proj $S^{N}$, the "quotient" of $M$ by the $N$-action. Any $B$-invariant subvariety $X$ of $M$ maps to a $T_{\mathbf{C}}$-invariant subvariety $Y$ of $M_{\text {impl }}$, whose $T$-moment map image is equal to $\Delta(X)$. But the image of $Y$ is convex by Atiyah's theorem. Therefore $\Delta(X)$ is convex. It is possible to give a rigorous argument along these lines, but we will present a shorter and more direct proof.

(iii) In contrast to the $G$-invariant case, the intersection $\Phi(X) \cap \mathfrak{t}^{*}$ is not Weyl group invariant. We do not know how $\Phi(X)$ intersects the chambers of $\mathbf{t}^{*}$ other than $\mathfrak{t}_{+}^{*}$. See however Remark 3.4 for some partial information.

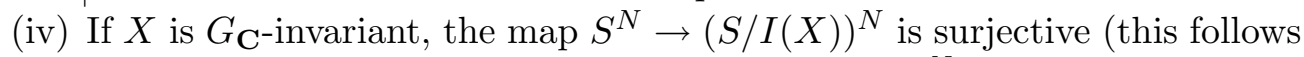
from the reductivity of $G_{\mathbf{C}}$ ) and thus $A(X)=(S / I(X))^{N}$. However, this may fail if $X$ is merely $B$-invariant, in which case the algebra $(S / I(X))^{N}$ may have undesirable properties.

Theorem 2.1 implies that $\Delta(\overline{B x})$ is a rational convex polytope for every point $x \in M$. For those points at which $B$ acts freely, a somewhat different description of these polytopes can be found in [10, Section 4.5], where we prove

2.3. Theorem. Let $x \in M$ and suppose that $B$ acts freely at $x$. Then

$$
\Delta(\overline{B x})=\mathfrak{t}_{+}^{*} \cap \bigcap_{n \in N} \Phi_{T}\left(\overline{T_{\mathbf{C}} n x}\right),
$$


where $\Phi_{T}: M \rightarrow \mathfrak{t}^{*}$ is the T-moment map, i.e. the composition of $\Phi$ with the projection $\mathfrak{g}^{*} \rightarrow \mathfrak{t}^{*}$.

(In [loc. cit., Theorem 4.5.1] $B$ denotes the negative Borel relative to the chamber $\mathfrak{t}_{+}^{*}$. The difference arises from the opposite sign convention for the moment map adopted there.) A similar result, for moment polytopes of $G_{\mathbf{C}}$-invariant subvarieties, was proved by Franz [8]. Notice that by Atiyah's theorem each of the sets $\Phi_{T}\left(\overline{T_{\mathbf{C}} n x}\right)$ is a convex polytope and only a finite number of distinct polytopes can occur in the intersection (3). Hence (3) is a convex polytope. However, our proof of Theorem 2.1 will be independent of Theorem 2.3.

The proof of Theorem 2.1 involves the following result, which is of interest in its own right:

2.4. Theorem. Let $X$ be a $B$-invariant irreducible closed subvariety of $M$ and let $i: X \rightarrow M$ be the inclusion map. Suppose $s \in S_{r \lambda, r}^{N}$ satisfies $i^{*} s \neq 0$. Let $x$ be a point on $X$ where $\left\|i^{*} s\right\|$ takes its maximum value. Then $\Phi(x)=\lambda$.

We mention a few consequences of Theorem 2.1. As in the torus and reductive cases, $\Delta(X)=\Delta(\overline{B x})$ for "most" $x$ in $X$ :

2.5. Corollary. Let $X$ be a B-invariant irreducible closed subvariety of $M$. The set $U$ consisting of all $x$ in $X$ for which $\Delta(X)=\Delta(\overline{B x})$ is nonempty and Zariski open in $X$.

Proof. It follows from Theorem 2.1 that

$$
U=\{x \in X \mid \mathcal{C}(X)=\mathcal{C}(\overline{B x})\} .
$$

Let $\lambda_{1}, \lambda_{2}, \ldots, \lambda_{l}$ be the vertices of $\mathcal{C}(X)$. For each $i$ put

$$
\begin{aligned}
U_{i}=\left\{x \in X \mid \lambda_{i}\right. & \in \mathcal{C}(\overline{B x})\} \\
& =\left\{x \in X \mid s(x) \neq 0 \text { for some } r>0 \text { and some } s \in S_{r \lambda_{i}, r}^{N}\right\} .
\end{aligned}
$$

It follows from (4) that $U=U_{1} \cap U_{2} \cap \cdots \cap U_{l}$. Each $U_{i}$ is nonempty and Zariski open, $X$ is irreducible, and therefore $U$ is nonempty and Zariski open.

By induction on the dimension this implies the following result.

2.6. Corollary. The collection of polytopes $\Delta(X)$, where $X$ ranges over all $B$ invariant irreducible closed subvarieties of $M$, is finite.

Corollary 2.5 applies to $G_{\mathbf{C}}$-invariant subvarieties $X$. In particular:

2.7. Corollary. The collection of $x \in M$ for which $\Delta(\overline{B x})=\Delta\left(\overline{G_{\mathbf{C}} x}\right)=\Delta(M)$ is nonempty and Zariski open. 
We refer to the following properties as the lower semi-continuity of the function $x \mapsto \Delta(\overline{B x})$.

2.8. Corollary. Let $x$ be an arbitrary point of $M$ and define

$$
\begin{gathered}
M_{\geq x}=\{y \in M \mid \Delta(\overline{B y}) \supseteq \Delta(\overline{B x})\}, \\
M_{\leq x}=\{y \in M \mid \Delta(\overline{B y}) \subseteq \Delta(\overline{B x})\}, \\
M_{x}=\{y \in M \mid \Delta(\overline{B y})=\Delta(\overline{B x})\} .
\end{gathered}
$$

Then $M_{\geq x}$ is open, $M_{\leq x}$ is closed, and $M_{x}$ is locally closed in the Zariski topology on $M$.

Proof. Theorem 2.1 shows that $M_{\geq x}=\{y \in M \mid \mathcal{C}(\overline{B y}) \supseteq \mathcal{C}(\overline{B x})\}$ (and analogous identities for $M_{\leq x}$ and $\left.M_{x}\right)$. The proof that $M_{\geq x}$ is open is now similar to the proof of Corollary 2.5. (Use the vertices of $\mathcal{C}(\overline{B x})$ instead of those of $\mathcal{C}(X)$.) The closedness of $M_{\leq x}$ then follows from the observation that

$$
M_{\leq x}=M-\bigcup\left\{M_{\geq y} \mid y \notin M_{\leq x}\right\} .
$$

Finally, $M_{x}=M_{\geq x} \cap M_{\leq x}$, so $M_{x}$ is locally closed.

\section{ThE NORM OF AN $N$-INVARIANT HOLOMORPHIC SECTION}

In this section we will prove Theorem 2.4 and part of Theorem 2.1. The group $G_{\mathbf{C}}$ acts complex linearly on the space of smooth global sections $C^{\infty}(M, L)$. Therefore $\mathcal{L}(\sqrt{-1} \xi) s=\sqrt{-1} \mathcal{L}(\xi) s$ for all $s \in C^{\infty}(M, L)$. Let $J$ be the complex structure on $M$. Since $G$ acts holomorphically on $M$, we have $(\sqrt{-1} \xi)_{M}=J \xi_{M}$ for all $\xi \in \mathfrak{g}$. Now suppose $s$ is a holomorphic section of $L$. Then, by the Cauchy-Riemann equation, $\nabla\left(J \xi_{M}\right) s=\sqrt{-1} \nabla\left(\xi_{M}\right) s$. Thus from (2) we get

$$
\mathcal{L}(\sqrt{-1} \xi) s-\nabla\left((\sqrt{-1} \xi)_{M}\right) s=\sqrt{-1}\left(\mathcal{L}(\xi)-\nabla\left(\xi_{M}\right)\right) s=-2 \pi \phi(\xi) s .
$$

For arbitrary $\xi \in \mathfrak{g}_{\mathbf{C}}$ let us write $\operatorname{Re} \xi=(\xi+\bar{\xi}) / 2$ and $\operatorname{Im} \xi=(\xi-\bar{\xi}) / 2 \sqrt{-1}$, where $\bar{\xi}$ is the complex conjugate of $\xi$ relative to the compact real form $\mathfrak{g}$ of $\mathfrak{g}_{\mathbf{C}}$. Also let us write $\phi_{\mathbf{C}}: \mathfrak{g}_{\mathbf{C}} \rightarrow C^{\infty}(M, \mathbf{C})$ for the complexification of the moment map $\phi: \mathfrak{g} \rightarrow C^{\infty}(M, \mathbf{R})$, given by $\phi_{\mathbf{C}}(\xi)=\phi(\operatorname{Re} \xi)+\sqrt{-1} \phi(\operatorname{Im} \xi)$ for $\xi \in \mathfrak{g}_{\mathbf{C}}$. Then (2) and (5) imply

$$
\mathcal{L}(\xi) s-\nabla\left(\xi_{M}\right) s=2 \pi \sqrt{-1} \phi_{\mathbf{C}}(\xi) s
$$

for all $\xi \in \mathfrak{g}_{\mathbf{C}}$ and all $s \in \Gamma(M, L)$. Now suppose that, for some $\xi \in \mathfrak{g}_{\mathbf{C}}, s$ is an eigensection for the operator $\mathcal{L}(\xi)$ with eigenvalue $c \in \mathbf{C}$. Then

$$
\nabla\left(\xi_{M}\right) s=\left(c-2 \pi \sqrt{-1} \phi_{\mathbf{C}}(\xi)\right) s,
$$

and hence

$$
\mathcal{L}\left(\xi_{M}\right)\|s\|^{2}=2 \operatorname{Re}\left\langle\nabla\left(\xi_{M}\right) s \mid s\right\rangle=(2 \operatorname{Re} c+4 \pi \phi(\operatorname{Im} \xi))\|s\|^{2},
$$


where $\langle\cdot \mid \cdot\rangle$ denotes the Hermitian inner product on $L$ and $\|\cdot\|$ the associated (pointwise) norm. This proves the following assertion.

3.1. Proposition. Let $s$ be a global holomorphic section of $L$. Let $\mathfrak{k}$ be a complex Lie subalgebra of $\mathfrak{g}_{\mathbf{C}}$ and suppose $s$ transforms under $\mathfrak{k}$ according to a character $\chi: \mathfrak{k} \rightarrow \mathbf{C}$. Then

$$
\mathcal{L}\left(\xi_{M}\right)\|s\|^{2}=(2 \operatorname{Re} \chi(\xi)+4 \pi \phi(\operatorname{Im} \xi))\|s\|^{2}
$$

for all $\xi \in \mathfrak{k}$.

3.2. Remarks. (i) The same formula holds for sections of $L^{r}$, provided we replace $\phi$ with $r \phi$.

(ii) The formula holds not only for global holomorphic sections, but also for holomorphic sections defined over a $\mathfrak{k}$-invariant analytic subvariety of $M$.

There are two important special cases of this identity. First let $\mathfrak{k}=\mathfrak{g}_{\mathbf{C}}$ and $\chi=0$. Then we get, for every $G$-invariant holomorphic section $s$ and $\xi \in \mathfrak{g}$,

$$
\mathcal{L}\left((\sqrt{-1} \xi)_{M}\right)\|s\|^{2}=4 \pi \phi(\xi)\|s\|^{2} .
$$

Recall that $x \in M$ is semistable if there exist $r>0$ and $s \in \Gamma\left(M, L^{r}\right)^{G}$ such that $s(x) \neq 0$. Let $x$ be semistable and $Y=\overline{G_{\mathbf{C}} x}$. If $y \in Y$ is a point where $\|s \mid Y\|$ attains its maximum, then (6) implies $\Phi(y)=0$. This proves the inclusion " $\subseteq$ " of the following theorem, in which $M^{\text {ss }}$ denotes the set of semistable points.

3.3. Theorem. $M^{\mathrm{ss}}=\left\{x \in M \mid 0 \in \Phi\left(\overline{G_{\mathbf{C}} x}\right)\right\}$.

The reverse inclusion "?" of this theorem was proved in [12, Theorem 5.6] under the assumption that 0 is a regular value of $\Phi$, in [13, Theorem 8.10] and in [16, Section 2] under the assumption that $\omega$ is the restriction to $M$ of the FubiniStudy symplectic form and $\Phi$ the standard moment map, and in [17, Proposition $2.4]$ in the general case.

A second important case of Proposition 3.1 is when $\mathfrak{k}=\mathfrak{b}$, the Borel subalgebra of $\mathfrak{g}_{\mathbf{C}}$. Let pr: $\mathfrak{b} \rightarrow \mathfrak{g}$ be the restriction to $\mathfrak{b}$ of the real linear projection $\operatorname{Im}: \mathfrak{g}_{\mathbf{C}} \rightarrow$ $\mathfrak{g}$. Let $R$ be the root system of $(G, T)$ and $R_{+}$the set of positive roots. Then

$$
\mathfrak{b}=\mathfrak{t}_{\mathbf{C}} \oplus \mathfrak{n}=\mathfrak{t} \oplus \sqrt{-1} \mathfrak{t} \oplus \bigoplus_{\alpha \in R_{+}} \mathfrak{g}_{\alpha}
$$

Hence pr sends $\mathfrak{t}$ to 0 and maps $\sqrt{-1} \mathfrak{t}$ bijectively onto $\mathfrak{t}$. Since $\overline{\mathfrak{g}}_{\alpha}=\mathfrak{g}_{-\alpha}$, pr maps $\mathfrak{n}$ bijectively onto the Ad-invariant complement

$$
\mathfrak{h}=\bigoplus_{\alpha \in R_{+}}\left(\mathfrak{g}_{\alpha} \oplus \mathfrak{g}_{-\alpha}\right) \cap \mathfrak{g}
$$

of $\mathfrak{t}$. In particular, pr is onto. 
The space of global sections is a $B$-module, so if $s \in \Gamma(M, L)$ is an eigensection for $\mathfrak{b}$ with character $\chi: \mathfrak{b} \rightarrow \mathbf{C}$, then $\chi$ exponentiates to a character of $B$. The two restriction maps

$$
\operatorname{Hom}\left(B, \mathbf{C}^{\times}\right) \longrightarrow \operatorname{Hom}\left(T_{\mathbf{C}}, \mathbf{C}^{\times}\right) \longrightarrow \operatorname{Hom}(T, \mathbf{U}(1))=\Lambda
$$

are isomorphisms, because $B=T_{\mathbf{C}} N$ and $[B, B]=N$ is unipotent. Therefore

$$
\chi(\xi)=2 \pi \sqrt{-1} \lambda\left(\xi_{1}+\sqrt{-1} \xi_{2}\right)=2 \pi \sqrt{-1} \lambda\left(\xi_{1}\right)-2 \pi \lambda\left(\xi_{2}\right)
$$

for a unique $\lambda \in \Lambda$. (Here we decompose $\xi \in \mathfrak{b}$ as $\xi_{1}+\sqrt{-1} \xi_{2}+\xi_{3}$ with $\xi_{1}$, $\xi_{2} \in \mathfrak{t}$ and $\xi_{3} \in \mathfrak{n}$.) This implies that $\operatorname{Re} \chi(\xi)=-2 \pi \lambda\left(\xi_{2}\right)$. Extending $\lambda$ to an element of $\mathfrak{g}^{*}$ by setting $\lambda=0$ on $\mathfrak{h}$, and recalling that $\operatorname{pr} \xi_{3} \in \mathfrak{h}$, we obtain $\operatorname{Re} \chi(\xi)=-2 \pi \lambda(\operatorname{pr} \xi)$. Thus Proposition 3.1 takes the form

$$
\mathcal{L}\left(\xi_{M}\right)\|s\|^{2}=4 \pi(-\lambda(\operatorname{pr} \xi)+\phi(\operatorname{pr} \xi))\|s\|^{2}
$$

for all $\xi \in \mathfrak{b}$ and all $N$-invariant holomorphic sections $s$ of weight $\lambda$. Similarly, for $s \in S_{r \lambda, r}^{N}$ we have

$$
\mathcal{L}\left(\xi_{M}\right)\|s\|^{2}=4 \pi r(-\lambda(\operatorname{pr} \xi)+\phi(\operatorname{pr} \xi))\|s\|^{2} .
$$

Proof of Theorem 2.4. Let $x$ be a point in $X$ where the function $\left\|i^{*} s\right\|$ attains its maximum. Then $\mathcal{L}\left(\xi_{M}\right)\|s\|^{2}=0$ for all $\xi \in \mathfrak{b}$, since $X$ is $B$-invariant. Moreover, $s(x) \neq 0$ because $i^{*} s \neq 0$. Hence $\phi(\operatorname{pr} \xi)(x)=\lambda(\operatorname{pr} \xi)$ by $(7)$. Since $\operatorname{pr}: \mathfrak{b} \rightarrow \mathfrak{g}$ is surjective, this implies $\Phi(x)=\lambda$.

3.4. Remark. Although for large $r$ the restriction map $\Gamma\left(M, L^{r}\right) \rightarrow \Gamma\left(X, L^{r}\right)$ is surjective, the induced map on $N$-invariants, $\Gamma\left(M, L^{r}\right)^{N} \rightarrow \Gamma\left(X, L^{r}\right)^{N}$, may not be. (See Remark 2.2(iv).) Nevertheless, it follows from Remark 3.2(ii) that Theorem 2.4 is also valid for sections $s \in \Gamma\left(X, L^{r}\right)^{N}$ that do not extend to global $N$-invariants. Hence, if $r \lambda \in \Lambda$ is any weight of $T_{\mathbf{C}}$ occurring in $\Gamma\left(X, L^{r}\right)^{N}$, whether dominant or not, then $\lambda \in \Phi(X)$. This proves the following assertion: let $A^{\prime}(X) \supseteq A(X)$ be the $\Lambda \times \mathbf{N}$-graded algebra $(S / I(X))^{N}$ and put

$$
\mathcal{C}^{\prime}(X)=\left\{\lambda \in \Lambda \otimes \mathbf{Q} \mid A^{\prime}(X)_{r \lambda, r} \neq 0 \text { for some } r>0\right\} .
$$

Then $\mathcal{C}^{\prime}(X)$ is convex over $\mathbf{Q}$ and is a subset of $\Phi(X) \cap \mathfrak{t}^{*}$.

Theorems 2.4 and 3.3 enable us to establish one half of Theorem 2.1.

3.5. Theorem. $\mathcal{C}(X)$ is equal to the set of rational points in $\Delta(X)$.

Proof. The inclusion $\mathcal{C}(X) \subseteq \Delta(X) \cap(\Lambda \otimes \mathbf{Q})$ follows from Theorem 2.4. Now let $\lambda \in \Delta(X)$ be a rational point. Then $\lambda=\Phi(x)$ for some $x \in X$, and $r_{0} \lambda \in \Lambda_{+}$ for some positive integer $r_{0}$. Without loss of generality we may replace $r_{0} \lambda$ with $\lambda, L$ with $L^{r_{0}}$, and $\Phi$ with $r_{0} \Phi$. Then $\Phi(x)=\lambda \in \Lambda_{+}$. Let

$$
\mathfrak{p}_{\lambda}=\mathfrak{b} \oplus \bigoplus\left\{\mathfrak{g}_{-\alpha} \mid \alpha \in R_{+}, \lambda\left(\alpha^{\vee}\right)=0\right\}
$$


be the parabolic subalgebra of $\mathfrak{g}_{\mathbf{C}}$ associated with $\lambda$ and $P_{\lambda}$ the parabolic subgroup of $G_{\mathbf{C}}$ generated by $\exp \mathfrak{p}_{\lambda}$. Let $Y=G_{\mathbf{C}} / P_{\lambda}$ be the corresponding flag variety and $L_{-\lambda}=\left(G_{\mathbf{C}} \times \mathbf{C}_{-\lambda}\right) / P_{\lambda}$ the Hermitian holomorphic line bundle on $Y$ determined by $-\lambda$. Here $\mathbf{C}_{-\lambda}$ is the one-dimensional $P_{\lambda}$-module with character $-\lambda$. The curvature form $\omega_{-\lambda}$ of $L_{-\lambda}$ is Kähler and a moment map $\Phi_{-\lambda}$ for the $G$-action is found, as in (1), by composing the maps

$$
Y=G_{\mathbf{C}} / P_{\lambda} \longrightarrow G / G_{\lambda} \longrightarrow \mathfrak{g}^{*} .
$$

Here $G_{\lambda}=P_{\lambda} \cap G$ is the centralizer of $\lambda$ (considered as an element of $\mathfrak{g}^{*}$ ), the first map is the inverse of the diffeomorphism $G / G_{\lambda} \rightarrow Y$ induced by the inclusion $G \rightarrow G_{\mathbf{C}}$, and the second map is defined by $g G_{\lambda} \mapsto-g \lambda$. Let us consider the product $M^{\prime}=M \times Y$ with the Kähler form $\omega^{\prime}=\omega+\omega_{-\lambda}$ as a Hamiltonian $G$ manifold with the diagonal $G$-action. The moment map on $M^{\prime}$ is $\Phi^{\prime}=\Phi+\Phi_{-\lambda}$, so from $\Phi(x)=\lambda$ we see that $\Phi^{\prime}\left(x, 1 P_{\lambda}\right)=0$. Now $\omega^{\prime}$ is the curvature form of the Hermitian holomorphic line bundle $L^{\prime}=L \otimes L_{-\lambda}$ and so, by Theorem 3.3, there exist $r>0$ and $s^{\prime} \in \Gamma\left(M^{\prime},\left(L^{\prime}\right)^{r}\right)^{G}$ satisfying

$$
s^{\prime}\left(x, 1 P_{\lambda}\right) \neq 0 .
$$

By the Borel-Weil theorem, $\Gamma\left(Y, L_{-\lambda}\right) \cong V_{\lambda}^{*}$, the dual of the irreducible $G$-module $V_{\lambda}$ with highest weight $\lambda$. Accordingly, the Künneth formula yields isomorphisms of $T_{\mathbf{C}}$-modules

$$
\begin{aligned}
\Gamma\left(M^{\prime},\left(L^{\prime}\right)^{r}\right)^{G} \cong\left(\Gamma\left(M, L^{r}\right) \otimes\right. & \left.\Gamma\left(Y, L_{-r \lambda}\right)\right)^{G} \cong\left(\Gamma\left(M, L^{r}\right) \otimes V_{r \lambda}^{*}\right)^{G} \\
& \cong \operatorname{Hom}\left(V_{r \lambda}, \Gamma\left(M, L^{r}\right)\right)^{G} \cong \Gamma\left(M, L^{r}\right)_{r \lambda}^{N}=S_{r \lambda, r}^{N} .
\end{aligned}
$$

A concrete isomorphism $\rho: \Gamma\left(M^{\prime},\left(L^{\prime}\right)^{r}\right)^{G} \rightarrow S_{r \lambda, r}^{N}$ is given by restricting invariant sections of $\left(L^{\prime}\right)^{r}$ to the first factor $M \cong M \times\left\{1 P_{\lambda}\right\}$, as indicated in the pullback diagram

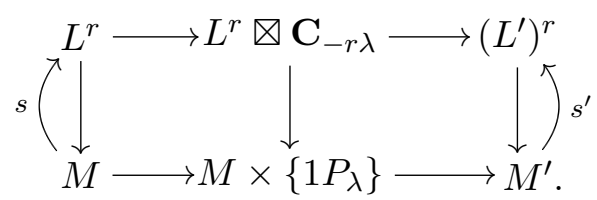

Explicitly, if $s^{\prime} \in \Gamma\left(M^{\prime},\left(L^{\prime}\right)^{r}\right)^{G}$, then $\rho\left(s^{\prime}\right)$ is the section of $L^{r}$ defined by $\rho\left(s^{\prime}\right)(y) \otimes 1=s^{\prime}\left(y, 1 P_{\lambda}\right)$ for all $y \in M$. It is easy to check that $\rho\left(s^{\prime}\right)$ is $N$ invariant and transforms according to $r \lambda$ under the $T_{\mathbf{C}}$-action. Conversely, a section $s \in S_{r \lambda, r}^{N}$ can be extended in a unique $G_{\mathbf{C}^{-}}$equivariant way to a global section $\epsilon(s)$ of $\left(L^{\prime}\right)^{r}$, given by the formula $\epsilon(s)\left(y, g P_{\lambda}\right)=g\left(s\left(g^{-1} y\right) \otimes 1\right)$ for $y \in M$ and $g \in G_{\mathbf{C}}$. It is easy to verify that $\epsilon(s)$ is well-defined and that the extension map $\epsilon: S_{r \lambda, r}^{N} \rightarrow \Gamma\left(M^{\prime},\left(L^{\prime}\right)^{r}\right)^{G}$ is the inverse of the restriction map $\rho$.

Taking $s^{\prime}$ as in (8) and putting $s=\rho\left(s^{\prime}\right)$ we find $s \in S_{r \lambda, r}^{N}$ satisfying $s(x) \neq 0$. Thus $\lambda \in \mathcal{C}(X)$. This proves $\Delta(X) \cap(\Lambda \otimes \mathbf{Q}) \subseteq \mathcal{C}(X)$. 


\section{Denseness of the RAtional Points}

In this section we will finish the proof of Theorem 2.1 by establishing the following result.

\subsection{Theorem. $\mathcal{C}(X)$ is dense in $\Delta(X)$.}

The proof is a variation on the proof of Theorem 3.5, namely a semistability argument involving a product $M \times Y_{n}$. However, for our present purposes $Y_{n}$ will not be a rational coadjoint orbit, but a member of a sequence $\left(Y_{n}\right)_{n \geq 1}$ of rational Kähler $G$-manifolds "converging" to an irrational coadjoint orbit.

This auxiliary sequence of spaces is manufactured as follows. Let $\lambda$ be any point in the closed Weyl chamber $\mathfrak{t}_{+}^{*}$. The chamber is a polyhedral cone and hence is a disjoint union of (relatively open) faces. Let $\sigma$ denote the unique face containing $\lambda$, let $\mathfrak{t}_{\sigma}^{*}$ be the linear span of $\sigma$ and let $\Lambda_{\sigma}$ be the lattice $\Lambda \cap \mathfrak{t}_{\sigma}^{*}$. The centralizer $G_{\sigma}=G_{\lambda}$ of $\sigma$ is a connected subgroup of $G$ containing the maximal torus $T$. The root system of $\left(G_{\sigma}, T\right)$ is the set $R_{\sigma}$ consisting of all roots $\alpha \in R$ such that $\lambda\left(\alpha^{\vee}\right)=0$, and so the complexification of the Lie algebra $\mathfrak{g}_{\sigma}$ has rootspace decomposition

$$
\left(\mathfrak{g}_{\sigma}\right)_{\mathbf{C}}=\mathfrak{t}_{\mathbf{C}} \oplus \bigoplus_{\alpha \in R_{\sigma}} \mathfrak{g}_{\alpha}
$$

From this we see that $\mathfrak{t}_{\sigma}^{*}$ is the annihilator in $\mathfrak{g}_{\sigma}^{*}$ of the ideal $\left[\mathfrak{g}_{\sigma}, \mathfrak{g}_{\sigma}\right]$ of $\mathfrak{g}_{\sigma}$, and therefore is canonically isomorphic to the dual of the Lie algebra $\mathfrak{t}_{\sigma}$ of the torus $T_{\sigma}=G_{\sigma} /\left[G_{\sigma}, G_{\sigma}\right]$. This implies that the canonical map

$$
\operatorname{Hom}\left(T_{\sigma}, \mathbf{U}(1)\right) \stackrel{\cong}{\longrightarrow} \operatorname{Hom}\left(G_{\sigma}, \mathbf{U}(1)\right) \hookrightarrow \operatorname{Hom}(T, \mathbf{U}(1))=\Lambda
$$

sends the weight lattice of $T_{\sigma}$ isomorphically onto the subgroup $\Lambda_{\sigma}$ of $\Lambda$. We will use this natural isomorphism to identify $\operatorname{Hom}\left(T_{\sigma}, \mathbf{U}(1)\right)$ with $\Lambda_{\sigma}$. Now let $\left(\Delta_{n}\right)_{n \geq 1}$ be any sequence of polytopes in $\mathfrak{t}^{*}$ subject to the following requirements:

(i) $\Delta_{n} \subseteq \sigma$ for all $n$;

(ii) $\Delta_{m} \subseteq \Delta_{n}$ for $m \geq n$;

(iii) $\bigcap_{n=1}^{\infty} \Delta_{n}=\{\lambda\}$;

(iv) for each $n$ there exists a positive integer $d_{n}$ such that the dilated polytope $d_{n} \Delta_{n}$ is a regular lattice polytope in $\mathfrak{t}_{\sigma}^{*}$ with respect to the lattice $\Lambda_{\sigma}$.

(Recall that for any free abelian group $F$ a regular lattice polytope is a subset $P$ of the vector space $F \otimes_{\mathbf{Z}} \mathbf{R}$ which is the convex hull of a finite subset of $F$ and each vertex $v$ of which has the property that the cone over $P$ with apex $v$ is spanned by a basis of $F$.) Let $\left(Y_{n}^{\circ}, \omega_{n}^{\circ}\right)$ be the symplectic toric $T_{\sigma}$-manifold associated with $\Delta_{n}$, as constructed by Delzant [7]. This is a compact Kähler manifold equipped with a Kählerian $T_{\sigma}$-action and a $T_{\sigma}$-moment map $\Phi_{n}^{\circ}$, whose image is exactly $\Delta_{n}$. The form $d_{n} \omega_{n}^{\circ}$ is integral and so there exists a unique $T$-equivariant Hermitian 
holomorphic line bundle $L_{n}^{\circ}$ over $Y_{n}^{\circ}$ whose curvature form is equal to $d_{n} \omega_{n}^{\circ}$. Since $T_{\sigma}$ is just the abelianization of $G_{\sigma}$, we can regard $Y_{n}^{\circ}$ as a Hamiltonian $G_{\sigma}$-manifold and $L_{n}^{\circ}$ as a $G_{\sigma}$-equivariant line bundle. We can boost $Y_{n}^{\circ}$ to a Hamiltonian $G$-manifold $Y_{n}$ by the familiar process of symplectic induction: put $Y_{n}=\left(T^{*} G \times Y_{n}^{\circ}\right) / / G_{\sigma}$, where $G_{\sigma}$ acts on $T^{*} G$ by right multiplication and by the given action on $Y_{n}^{\circ}$, and where the double slash indicates symplectic reduction at the zero level. The left $G$-action on $T^{*} G$ descends to a $G$-action on $Y_{n}$ and the $G_{\sigma}$-moment map $\Phi_{n}^{\circ}$ induces a $G$-equivariant map $\Phi_{n}: Y_{n} \rightarrow \mathfrak{g}^{*}$, which is a moment map for the $G$-action with respect to the reduced symplectic form $\omega_{n}$. The line bundle $L_{n}^{\circ}$ induces a Hermitian line bundle $L_{n}$ on $Y_{n}$ with curvature form $d_{n} \omega_{n}$ and, finally, the holomorphic structures on $Y_{n}^{\circ}$ and $L_{n}^{\circ}$ induce $G$-invariant holomorphic structures on $Y_{n}$, resp. $L_{n}$. (In fact, there are isomorphisms of complex $G_{\mathbf{C}^{-}}$manifolds

$$
Y_{n} \cong G_{\mathbf{C}} \times{ }^{P_{\lambda}} Y_{n}^{\circ} \quad \text { and } \quad L_{n} \cong G_{\mathbf{C}} \times{ }^{P_{\lambda}} L_{n}^{\circ},
$$

where the parabolic subgroup $P_{\lambda}$ acts on $Y_{n}^{\circ}$ and $L_{n}^{\circ}$ via the Levi factor $\left(G_{\lambda}\right)_{\mathbf{C}}$.) By construction, the $G$-manifold $Y_{n}$ has moment polytope $\Delta\left(Y_{n}\right)=\Phi_{n}\left(Y_{n}\right) \cap \mathfrak{t}_{+}^{*}=$ $\Delta_{n}$.

Proof of Theorem 4.1. Let $\lambda \in \Delta(X)$. We need to show that there exists a sequence $\left(\lambda_{n}\right)_{n \geq 1}$ in $\mathcal{C}(X)$ converging to $\lambda$. We choose a sequence of polytopes $\left(\Delta_{n}\right)_{n \geq 1}$ satisfying requirements (i)-(iv) above and we let $\left(Y_{n}\right)_{n \geq 1}$ be the corresponding sequence of Hamiltonian $G$-manifolds. Let $Y_{n}^{-}$denote the symplectic manifold opposite to $Y_{n}$ and let $M_{n}^{\prime}$ be the $G$-manifold $M \times Y_{n}^{-}$, furnished with the Kähler form $\omega_{n}^{\prime}=\omega-\omega_{n}$, the moment map $\Phi_{n}^{\prime}=\Phi-\Phi_{n}$ and the line bundle $L_{n}^{\prime}=L^{d_{n}} \otimes L_{n}^{*}$. Note that the equivariant curvature form of $L_{n}^{\prime}$ is equal to $d_{n}\left(\omega_{n}^{\prime}+\Phi_{n}^{\prime}\right)$. Choose any $x$ in $X$ such that $\Phi(x)=\lambda$. Since $\lambda \in \Delta_{n}=\Delta\left(Y_{n}\right)$, there exists $y_{n} \in Y_{n}$ such that $\Phi_{n}^{\prime}\left(x, y_{n}\right)=0$. Therefore Theorem 3.3 (applied to the symplectic manifold $\left.\left(M_{n}^{\prime}, d_{n} \omega_{n}^{\prime}\right)\right)$ guarantees the existence of a positive integer $r_{n}$ and a section $s_{n}^{\prime} \in \Gamma\left(M_{n}^{\prime},\left(L_{n}^{\prime}\right)^{r_{n}}\right)^{G}$ such that $s_{n}^{\prime}\left(x, y_{n}\right) \neq 0$. Let $p_{1}: M_{n}^{\prime} \rightarrow M$ and $p_{2}: M_{n}^{\prime} \rightarrow Y_{n}$ denote the Cartesian projections. The natural maps

$$
\begin{aligned}
\Gamma\left(M_{n}^{\prime},\left(L_{n}^{\prime}\right)^{r_{n}}\right) \stackrel{\cong}{\cong} \operatorname{Hom}\left(p_{2}^{*} L_{n}^{r_{n}}, p_{1}^{*} L^{d_{n} r_{n}}\right) & \\
& \cong \operatorname{Mom}\left(\Gamma\left(Y_{n}, L_{n}^{r_{n}}\right), \Gamma\left(M, L^{d_{n} r_{n}}\right)\right)
\end{aligned}
$$

are $G$-equivariant isomorphisms. Hence, by Schur's lemma, there exist a dominant weight $\mu$ and direct summands $E_{\mu}$ and $F_{\mu}$ of the $G$-modules $\Gamma\left(M, L^{d_{n} r_{n}}\right)$, resp. $\Gamma\left(Y_{n}, L_{n}^{r_{n}}\right)$, such that $E_{\mu}$ and $F_{\mu}$ are irreducible of highest weight $\mu$ and such that the image of $s_{n}^{\prime}$ in $\operatorname{Hom}\left(F_{\mu}, E_{\mu}\right)^{G}$ does not vanish at the point $\left(x, y_{n}\right)$. Therefore, if $s_{E}$ and $s_{F}$ are highest weight vectors in $E_{\mu}$, resp. $F_{\mu}$, we have $s_{E}(x) \neq 0$ and $s_{F}\left(y_{n}\right) \neq 0$. This shows that the point $\mu / r_{n}$ is contained in both $\mathcal{C}\left(Y_{n}, L_{n}\right)$ 
and $\mathcal{C}\left(X, L^{d_{n}}\right)$. In particular, by Theorem 3.5,

$$
\mu / r_{n} \in \mathcal{C}\left(Y_{n}, L_{n}\right) \subseteq \Delta\left(Y_{n}, d_{n} \omega_{n}\right),
$$

and hence $\mu / r_{n} \in d_{n} \Delta_{n}$, since $\Delta\left(Y_{n}, \omega_{n}\right)=\Delta_{n}$. We conclude that the point $\lambda_{n}=\mu / d_{n} r_{n}$ is contained in $\Delta_{n} \cap \mathcal{C}(X, L)$. Requirements (ii)-(iii) now ensure that $\lim _{n \rightarrow \infty} \lambda_{n}=\lambda$.

A useful corollary of these results is the following extension of Theorem 3.3. Let us call a point $x \in M$ unipotently semistable if there exist $r>0$ and $s \in$ $\Gamma\left(M, L^{r}\right)^{N}$ such that $s(x) \neq 0$, in other words, if the set $\mathcal{C}(\overline{B x})$ is nonempty. By Theorem 4.1, $\Delta(\overline{B x})$ is the closure of $\mathcal{C}(\overline{B x})$, so unipotent semistability can be characterized thus:

4.2. Theorem. A point $x$ is unipotently semistable if and only if $\Delta(\overline{B x})$ is nonempty.

\section{5. "SCHUBERT" VARIETIES}

In this section we will describe some subvarieties of $M$ to which Theorem 2.1 applies. Let $M_{1}^{T}, M_{2}^{T}, \ldots, M_{k}^{T}$ be the connected components of the $T$-fixed point set $M^{T}$. For $j=1,2, \ldots, k$, let $\alpha_{1 j}, \alpha_{2 j}, \ldots, \alpha_{c(j), j}$ be the nonzero weights of the isotropy representation of the maximal torus $T$ on the tangent space at any point of $M_{j}^{T}$, where $c(j)$ is the complex codimension of $M_{j}^{T}$. Let $\mathcal{P}$ be the complement of the rational hyperplane arrangement defined by this collection of weights:

$$
\mathcal{P}=\left\{\xi \in \mathfrak{t} \mid \alpha_{i j}(\xi) \neq 0 \text { for all } j=1,2, \ldots, k \text { and } i=1,2, \ldots, c(j)\right\} .
$$

The connected components of $\mathcal{P}$ are the action chambers of $\tau$. We fix, once and for all, an action chamber $\mathcal{P}_{+}$which intersects the Weyl chamber $\mathfrak{t}_{+}$. Pick an element $\xi \in \mathcal{P}_{+}$and put $\eta=\sqrt{-1} \xi \in \sqrt{-1} \mathrm{t}$. For each $y \in M$ the limit $x=\lim _{t \rightarrow-\infty} \exp (t \eta) y$ exists and is fixed under $\xi$. Since $\xi \in \mathcal{P}$, this implies that $x \in M^{T}$. The unstable manifold of $M_{j}^{T}$ is defined as the set

$$
W_{j}=\left\{y \in M \mid \lim _{t \rightarrow-\infty} \exp (t \eta) y \in M_{j}^{T}\right\} .
$$

Generalizing results of Białynicki-Birula [2, 3], Carrell and Sommese [5, 6] showed that $W_{j}$ is a complex submanifold of $M$, whose closure $X_{j}=\bar{W}_{j}$ is an analytic subvariety in which $W_{j}$ is Zariski open. Moreover, the map $\pi_{j}: W_{j} \rightarrow M_{j}^{T}$ which sends $y$ to $\lim _{t \rightarrow-\infty} \exp (t \eta) y$ is a holomorphic fibration, the fibres of which are complex affine spaces. Let us call $X_{j}$ a generalized Schubert variety of $M$. The varieties $W_{j}$ and $X_{j}$ and the fibration $\pi_{j}$ are defined by making a choice of an element $\xi$ of the action chamber; however, one can show that they don't depend on $\xi$ but only on the action chamber. (See e.g. [10, Section 3.6].) In particular, we may, and will, assume that $\xi \in \mathcal{P}_{+} \cap \operatorname{Int} \mathfrak{t}_{+}$. Then the parabolic subgroup associated with $\xi$ is equal to $B$, which implies the following result. 
5.1. Proposition. Let $1 \leq j \leq k$. Then

(i) the unstable manifold $W_{j}$ and the generalized Schubert variety $X_{j}$ are $B$-invariant;

(ii) the map $\pi_{j}$ is B-invariant;

(iii) for every $x \in M_{j}^{T}$ the fibre $W_{j, x}=\pi_{j}^{-1}(x)$ and its closure $X_{j, x}=\bar{W}_{j, x}$ are B-invariant.

Proof. It follows immediately from the definition that the submanifold $W_{j}$ and the map $\pi_{j}$ are $T_{\mathbf{C}}$-invariant. Let

$$
\mathfrak{n}=\bigoplus_{\alpha \in R_{+}} \mathfrak{g}_{\alpha}
$$

be the decomposition of the Lie algebra of $N$ into weight spaces with respect to the $T$-action, $R_{+}$being the set of positive roots of the root system $R$ of $(G, T)$. Let $\zeta \in \mathfrak{n}$ and write $\zeta=\sum_{\alpha \in R_{+}} \zeta_{\alpha}$ with $\zeta_{\alpha} \in \mathfrak{g}_{\alpha}$. Then $\operatorname{Ad}(\exp t \eta) \zeta=\sum_{\alpha} e^{2 \pi \alpha(\eta) t} \zeta_{\alpha}$. Since $\xi \in \operatorname{Int} \mathfrak{t}_{+}$, we have $\alpha(\eta)>0$ and hence

$$
\zeta(t)=\operatorname{Ad}(\exp t \eta) \zeta \longrightarrow 0 \quad \text { and } \quad \exp \zeta(t) \longrightarrow 1
$$

as $t \rightarrow-\infty$. In particular, if $y \in W_{j}$ and $n=\exp \zeta \in N$,

$$
\exp (t \eta) n y=\exp (t \eta) n \exp (-t \eta) \exp (t \eta) y=\exp \zeta(t) \exp (t \eta) y \longrightarrow \pi_{j}(y)
$$

as $t \rightarrow-\infty$. This shows that $W_{j}$ and $\pi_{j}$ are $N$-invariant. Since $B=T_{\mathbf{C}} N$, both are $B$-invariant as well. It follows immediately that $X_{j}, W_{j, x}$ and $X_{j, x}$ are $B$-invariant.

This shows that one can apply Theorem 2.1 to the generalized Schubert varieties $X_{j}$ and their subvarieties $X_{j, x}$. As might be expected, for most $x$ in the fixed-point component $M_{j}^{T}$ the polytopes $\Delta\left(X_{j}\right)$ and $\Delta\left(X_{j, x}\right)$ are the same.

5.2. Corollary. Let $1 \leq j \leq k$. The set of all $x \in M_{j}^{T}$ such that $\Delta\left(X_{j}\right)=\Delta\left(X_{j, x}\right)$ is a nonempty Zariski open subset of $M_{j}^{T}$.

Proof. Define

$$
U=\left\{y \in W_{j} \mid \Delta(\overline{B y})=\Delta\left(X_{j}\right)\right\} \quad \text { and } \quad C=\left\{x \in M_{j}^{T} \mid \Delta\left(X_{j, x}\right)=\Delta\left(X_{j}\right)\right\} .
$$

We claim that

$$
\pi_{j}(U)=C .
$$

Indeed, let $y \in U$ and put $x=\pi_{j}(y)$. Then $\overline{B y}$ is contained in $X_{j, x}$ since $X_{j, x}$ is $B$-invariant, and therefore

$$
\Delta\left(X_{j}\right)=\Delta(\overline{B y}) \subseteq \Delta\left(X_{j, x}\right) \subseteq \Delta\left(X_{j}\right),
$$

i.e. $x \in C$. This shows that $\pi_{j}(U)$ is a subset of $C$. Conversely, let $x \in C$. By Corollary 2.5, there exists $y \in W_{j, x}$ such that $\Delta(\overline{B y})=\Delta\left(X_{j, x}\right)$. Hence $\Delta(\overline{B y})=$ 
$\Delta\left(X_{j}\right)$, i.e. $y \in U$. This shows that $C$ is contained in $\pi_{j}(U)$, which proves (9). By Corollary 2.5, $U$ is nonempty and Zariski open in $W_{j}$ and therefore, by a theorem of Chevalley, its image $\pi_{j}(U)=C$ is constructible in $M_{j}^{T}$. Furthermore, $C$ is open in the classical topology, since $\pi_{j}$ is a submersion. Hence $C$ is Zariski open.

However, frequently the polytopes $\Delta\left(X_{j}\right)$ are not very exciting. More interesting polytopes turn up when we restrict the $G$-action on $M$ to a subgroup. Particularly interesting is the case of the flag variety $M=G_{\mathbf{C}} / B$. Here the $W_{j}$ 's are just the $B$-orbits in $M$, so the $X_{j}$ 's are the classical Schubert varieties. Let us now view $M$ as a $G_{1}$-space with respect to a closed connected subgroup $G_{1}$ of $G$. Let us suppose $B$ is chosen in such a way that it contains a Borel subgroup $B_{1}$ of $\left(G_{1}\right)_{\mathbf{C}}$, so that the $X_{j}$ 's are $B_{1}$-invariant. If $G_{1}$ is the maximal torus $T$ of $G$, one gets from Theorem 2.1 Atiyah's Theorem 1.4, and if one takes $G_{1}$ to be an arbitrary closed subgroup of $G$ one gets a nonabelian version of this theorem.

\section{REFERENCES}

1. M. Atiyah, Convexity and commuting Hamiltonians, Bull. London Math. Soc. 14 (1982), no. 1, 1-15. MR 83e:53037

2. A. Białynicki-Birula, Some theorems on actions of algebraic groups, Ann. of Math. (2) 98 (1973), 480-497. MR 51 \#3186

3. $\_$Some properties of the decompositions of algebraic varieties determined by actions of a torus, Bull. Acad. Polon. Sci. Sér. Sci. Math. Astronom. Phys. 24 (1976), no. 9, 667-674. MR 0453766 (56 \#12020)

4. M. Brion, Sur l'image de l'application moment, Séminaire d'algèbre Paul Dubreil et MariePaule Malliavin (Paris, 1986) (M.-P. Malliavin, ed.), Lecture Notes in Mathematics, vol. 1296, Springer-Verlag, Berlin, 1987, pp. 177-192. MR 89i:32062

5. J. Carrell and A. Sommese, C ${ }^{*}$-actions, Math. Scand. 43 (1978/79), no. 1, 49-59, Correction, ibid. 53, no. 1 (1983), 32. MR 523824 (80h:32053)

6. Some topological aspects of $\mathbf{C}^{*}$ actions on compact Kaehler manifolds, Comment. Math. Helv. 54 (1979), no. 4, 567-582. MR 552677 (80m:32032)

7. T. Delzant, Hamiltoniens périodiques et images convexes de l'application moment, Bull. Soc. Math. France 116 (1988), no. 3, 315-339. MR 90b:58069

8. M. Franz, Moment polytopes of projective G-varieties and tensor products of symmetric group representations, J. Lie Theory 12 (2002), no. 2, 539-549. MR 1923785 (2003j:20077)

9. V. Guillemin, L. Jeffrey, and R. Sjamaar, Symplectic implosion, Transform. Groups. 7 (2002), no. 2, 155-184. MR 2003b:53090

10. V. Guillemin and R. Sjamaar, Convexity properties of Hamiltonian group actions, CRM Monograph Series, vol. 26, American Mathematical Society, Providence, RI, 2005.

11. V. Guillemin and S. Sternberg, Convexity properties of the moment mapping, Invent. Math. 67 (1982), no. 3, 491-513. MR 83m:58037

12. 67 (1982), no. 3, 515-538. MR 83m:58040

13. F. Kirwan, Cohomology of quotients in symplectic and algebraic geometry, Mathematical Notes, vol. 31, Princeton University Press, Princeton, N.J., 1984. MR 86i:58050 
14. B. Kostant, Quantization and unitary representations. I. Prequantization, Lectures in Modern Analysis and Applications, III (Washington, D.C.) (C. T. Taam, ed.), Lecture Notes in Mathematics, vol. 170, Springer-Verlag, Berlin, 1970, pp. 87-208. MR 45 \#3638

15. H. Kraft, Geometrische Methoden in der Invariantentheorie, second revised ed., Aspekte der Mathematik, vol. D1, Vieweg, Braunschweig, 1985. MR 86j:14006

16. L. Ness, A stratification of the null cone via the moment map, Amer. J. Math. 106 (1984), no. $6,1281-1329$, with an appendix by D. Mumford. MR 86c:14010

17. R. Sjamaar, Holomorphic slices, symplectic reduction and multiplicities of representations, Ann. of Math. (2) 141 (1995), no. 1, 87-129. MR 96a:58098

18. T. Vust, Sur la théorie des invariants des groupes classiques, Ann. Inst. Fourier (Grenoble) 26 (1976), no. 1, 1-31. MR 0404280 (53 \#8082)

Victor Guillemin

Department of Mathematics

Massachusetts Institute of Technology

Cambridge, Massachusetts 02139-4307

E-mail:vwg@math.mit.edu

Reyer Sjamaar

Department of Mathematics

Cornell University, Ithaca

New York 14853-4201

E-mail: sjamaar@math.cornell.edu 